\title{
Bedside wireless lung ultrasound for the evaluation of COVID-19 lung injury in senior nursing home residents
}

\author{
Frank L. Dini ${ }^{1}$, Carlo Bergamini ${ }^{1}$, Aldo Allegrini ${ }^{1}$, Massimo Scopelliti ${ }^{1}$, Gianmarco Secco ${ }^{2,3}$, \\ Mario Miccoli ${ }^{4}$, Stefano Boni ${ }^{5}$, Raffaella Brigada ${ }^{5}$, Stefano Perlini2,3 \\ ${ }^{1}$ Medical Workforce, Department of Homeland Security; ${ }^{2}$ Emergency Department, Fondazione IRCCS Policlinico San \\ Matteo, Pavia; ${ }^{3}$ Department of Internal Medicine and Therapeutics, University of Pavia; ${ }^{4}$ Department of Clinical and \\ Experimental Medicine, University of Pisa, Pisa; ${ }^{5}$ Azienda per la Tutela della Salute, ATS, Pavia, Italy
}

\author{
Correspondence: Frank L. Dini, MD, ATS della Provincia di Pavia, Via \\ Indipendenza, 3, 27100, Pavia, Italy. \\ E-mail: franklloyddini@gmail.com
}

Key words: COVID-19 pneumonia, nasal-pharyngeal swabs, lung ultrasound, elderly.

Contributions:All the authors made a substantive intellectual contribution, performed part of the experiments. All the authors have read and approved the final version of the manuscript and agreed to be accountable for all aspects of the work.

Conflict of interest: The authors declare that they have no competing interests, and all authors confirm accuracy.

Availability of data and materials: All data generated or analyzed during this study are included in this published article.

Ethics approval and consent to participate: All procedures performed in studies involving human participants were in accordance with the ethical standards of the Institutional Research Committee and with the 1964 Helsinki declaration and its latest amendment. Written informed consent was obtained from a legally authorized representative(s) for anonymized patient information to be published in this article

Acknowledgments: We sincerely thank Doctor Mara Azzi, General Manager of the Azienda per la Tutela della Salute (ATS), Pavia, Italy, Doctor Angelo Borrelli and Doctor Gian Luca Sessa, from the Homeland Security Headquarter, Rome, Italy, for their fundamental contribution to direct and organize the Homeland Security COVID-19 Medical Task Force of the Pavia province. We thank all the staff nurses and physicians of the following nursing homes of the Pavia province: Casa di Riposo Delfinoni, Casorate Primo; Fondazione Galtrucco, Robbio; RSA Arcobaleno, Cava Manara; RSA Casa Serena, Cilavegna; RSA La Certosa, Certosa di Pavia; RSA Le Risaie, Marcignago, RSA Pezzani, Voghera; RSA San Gennaro, Varzi; RSA San Giuseppe, Belgioioso; RSA San Giuseppe, Dorno, RSA Sant'Anna, Garlasco; RSA Stradella-Broni, Stradella. Finally, we thank all the staff physicans of the Unità Speciali Continuità Assistenziale (USCA) for their valuable contribution to perform COVID-19 nasal-pharyngeal swamps.

Received for publication: 11 June 2020.

Accepted for publication: 10 July 2020.

${ }^{\mathbb{C}}$ Copyright: the Author(s), 2020

Licensee PAGEPress, Italy

Monaldi Archives for Chest Disease 2020; 90:1446

doi: 10.4081/monaldi.2020.1446

This article is distributed under the terms of the Creative Commons Attribution Noncommercial License (by-nc 4.0) which permits any noncommercial use, distribution, and reproduction in any medium, provided the original author(s) and source are credited.

\section{Abstract}

Lung Ultrasound (LUS) is regarded to be potentially useful to diagnose lung injury in older adults living in nursing homes with suspected COVID-19 pneumonia. We aimed at evaluating presence lung injury among senior nursing home residents by LUS performed with portable wireless scanner echography. The study population consisted of 150 residents with a mean age of 88 years ( $85 \%$ female) residing in 12 nursing homes in Northern Italy. Subjects had to have a history of recent onset of symptoms compatible with COVID-19 pneumonia or have been exposed to the contagion of patients carrying the disease. COVID-19 testing was performed with SARS-CoV-2 nasal-pharyngeal (NP) swabs. Positive subjects to LUS scanning were considered those with non-coascelent B-lines in $>3$ zones, coalescent B-lines in $>3$ zones and with iperdensed patchy non-consolidated lungs. Sixty-three percent had positive NP testing and 65\% had LUS signs of pulmonary injury. LUS had a sensitivity of $79 \%$ in predicting positive NP testing. Sixteen percent of residents tested negative for SARSCoV-2 carried the signs of COVID-19 lung injury at LUS. There were 92 patients $(61 \%)$ with current or recent symptoms. Positivity to LUS scanning was reported in $73 \%$ of residents with symptoms, while it was $53 \%$ in those without $(\mathrm{P}=0.016)$. A positive NP testing was observed in $66 \%$ of residents with symptoms and in $57 \%$ of those without $(\mathrm{P}=0.27)$. We conclude that assessment of LUS by portable wireless scanner echography can be profitability utilized to diagnose lung injury among senior nursing home residents with or without symptoms compatible with COVID-19 pneumonia.

\section{Introduction}

The SARS-CoV-2 pandemic is impacting the global population and community transmission had led to an overwhelming number of critically-ill patients [1]. Most of patients who are suffering from the COVID-19 disease exhibit signs of pulmonary injury that are evident from clinical as well as instrumental findings [2].

Studies have shown that COVID-19 lung disease causes worse outcomes and higher mortality in older patients, especially in those with co-morbidities, such as hypertension, heart disease, diabetes, chronic respiratory disease, and chronic kidney disease [3-6]. This may be especially harmful in the elderly population living in nursing homes for their baseline co-morbidities and exposures resulting from their congregate settings [7]. 
Lung ultrasound (LUS) is a noninvasive tool for the evaluation of lung disease and has the advantage of rapidity, repeatability and reproducibility. Therefore, it is increasingly used by physicians at bedside to complement the findings of physical examination $[8,9]$. With the introduction of portable echo wireless transthoracic scanners, LUS may become a valuable tool to investigate presence of pulmonary injury in the community, especially in patients living nursing home facilities, due to their frailty and pandemic vulnerability. These considerations have led us to design a study aiming at evaluating presence lung injury among senior nursing home residents by LUS performed by portable wireless scanner echography.

\section{Methods}

The study population consisted of consecutive subjects residing in nursing homes. Inclusion criteria were: patients institutionalized in residential age care facilities of Pavia province in Italy with a history of recent respiratory symptoms and/or fever or have been in contact with patients that have been previously tested positive to SARS-CoV-2 infection. Exclusion criteria included asymptomatic residents of nursing homes that were not exposed to the infection.

LUS was performed with a portable sector convex/linear wireless CERBERO (ATL, Milano, Italy) probes of $3.5 \mathrm{MHz}$ and 7.5$10 \mathrm{MHz}$ with no harmonic filter, connected with a tablet. Focus was placed on the pleural line, maximum depth was at $8-10 \mathrm{~cm}$. Mechanical index started from $0.7 \mathrm{~cm}$ and was reduced as further as possible. All devices were wrapped in single use plastic covers to reduce the risk of contamination and to facilitate the sterilization procedures. Patients were examined in supine or semi-recumbent position. Each hemithorax was divided by the anterior axillary line and posterior axillary line into three areas: anterior, lateral and posterior. Each of these zones was subsequently divided into upper and lower zones. The thorax was scanned in eight to twelve intercostal zones (four to six on each emithorax) depending on patient's condition. Pleural line, presence of pleural effusion and lung sliding were also assessed. We used a 4-level scoring system [9] to establish the severity of the patient's condition. Positive patients to LUS scanning were considered those with non-coascelent B-lines in $>3$ zones (score 1), coalescent B-lines in $>3$ zones (score 2) and with iperdensed non-consolidated state (score 3 ). A-lines or nonsignificant B-lines were classified as normal pattern (score 0).

COVID-19 testing was performed with SARS-CoV-2 nasalpharyngeal swabs (Universal Transport Medium, Copan Diagnostics, Inc., CA, USA) [10].

Statistical analyses were performed with 25.0 SPSS Package (IBM Corp., Armonk, NY, USA). Data were expressed as mean value \pm standard deviation or interquartile ranges (IQR) for continuous variables and percentages for categorical variables. Anderson-Darling test was performed to verify normality of distributions. Comparisons were made using Student's t-test and MannWhitney test. Chi-square test was utilized to compare categorical variables. Statistically significant differences were placed at $\mathrm{P}=0.05$. With positive testing for SARS-CoV-2 as reference standard, sensitivity, specificity, positive predictive value (PPV) and negative predicted value (NPV) of signs of lung injury at LUS, symptoms and oxygen saturation were evaluated. Cohen's kappa was calculated to measure the agreement levels between LUS and NP swab in the total group and in the two different subgroups. According with Landis and Koch interpretation [11], the following ranges of kappa values were considered: $<0$ : no agreement; $0.0-$
0.2: slight agreement; 0.21-0.40: fair agreement; 0.41-0.60: moderate agreement; 0.61-0.80: substantial agreement; 0.81-1.0: perfect agreement.

\section{Results}

The study population included 150 residents of 12 nursing home facilities of the province of Pavia (Lombardy; Italy) enrolled between April 2020 and May 2020. Mean age was 88 years (range: $72-106$ years; $85 \%$ female). Co-occurring diseases were present in almost all patients. History of hypertension was present in $61 \%$, history of kidney disease in $23 \%$, coronary artery disease in $17 \%$, other heart diseases in $27 \%$, cerebrovascular disease in $29 \%$, atrial fibrillation in $19 \%$, diabetes in $19 \%$, heart failure in $8 \%$ and chronic respiratory disease in $9 \%$.

Current or recent symptoms, from moderate to severe, including fever, respiratory symptoms (like cough and dyspnoea), and asthenia were reported in $61 \%$.

Ninety-eight $(65 \%)$ of patients had positive LUS findings. Among them, score 1 was reported in 36 patients, 32 were classified as score 2. Score 3 was observed in 30 . LUS showed pleural line abnormalities in $90 \%$ of patients, most of them were irregular and discontinued and sometimes fragmented. Sliding was preserved in all but two cases. Signs of pleural effusion were reported in 11 cases. Positivity to LUS scanning was reported in 67 patients (73\%) with symptoms, while it was $53 \%(\mathrm{n}=31)$ in those without $(\mathrm{P}=0.016)$.

Nasal-pharyngeal swabs for laboratory testing of SARS-CoV2 were collected in all study patients within a week from LUS assessment. Sixty-three percent of them $(n=94)$ resulted positive for SARS-CoV-2 infection. The positive rate of COVID-19 nasalpharyngeal sampling was $66 \%(\mathrm{n}=61)$ in patients with symptoms and $57 \%(\mathrm{n}=33)$ in those without $(\mathrm{P}=0.27)$. Table 1 summarizes the characteristics of the study patients among those presenting symptoms and/or fever and in those who were asymptomatic. Figure 1 shows percentages with score 1 , score 2 , and score 3 lung injury at LUS among patients with symptoms and those without symptoms.

In patients tested negative, $16 \%$ had positive LUS. Table 2 shows sensitivity, specificity, PPV and NPV of LUS abnormalities,

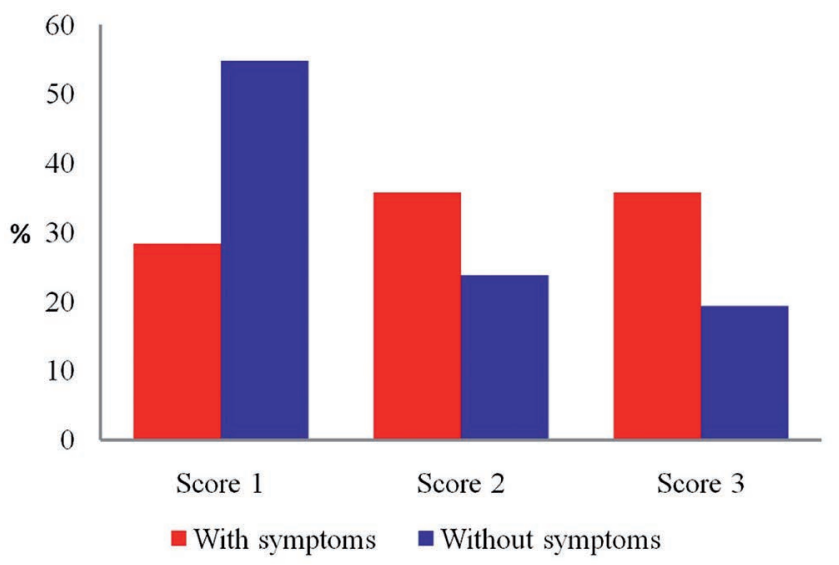

Figure 1. Lung ultrasound score in patients positive to lung ultrasound scanning divided according to presence or absence of prior or current symptoms and/or fever. A statistically significant difference $(P=0.011)$ was observed with Score 1 between symptomatic and asymptomatic subjects. 
symptoms and oxygen saturation in predicting positive laboratory testing of SARS-CoV-2 at nasal pharyngeal swabs. Signs of lung injury at LUS predicted positive laboratory testing with a sensitivity of $79 \%$ and a specificity of $57 \%$. Table 3 shows sensitivity, specificity, PPV and NPV of LUS, and oxygen saturation in predicting positivity of COVID-19 nasal-pharyngeal swabs in older residents with symptoms and in those without symptoms. As far as Cohen's kappa measures of the agreement between LUS and SARS-CoV-2 nasal-pharyngeal swabs is concerned, the coefficient was 0.36 in the total group, 0.34 in patients with symptoms and 0.37 in those who were asymptomatic.

\section{Discussion}

The results of the present study show that assessment of LUS by portable wireless scanner echography can be profitability utilized to diagnose lung injury among senior nursing home residents who manifested symptoms compatible with COVID-19 pneumonia and in those exposed to the contagion of patients bearing the disease. We found that $16 \%$ of patients tested negative for SARSCoV-2 carried the signs of COVID-19 lung injury at LUS.

Older adults living in residential homes are at increased risk of

Table 1. Characteristics of the study patients of the overall population and of groups divided according to presence or absence of prior or current symptoms and/or fever.

\begin{tabular}{|c|c|c|c|c|}
\hline Variable & $\begin{array}{l}\text { Overall } \\
(n=150)\end{array}$ & $\begin{array}{l}\text { With symptoms } \\
\qquad(\mathrm{n}=92)\end{array}$ & $\begin{array}{l}\text { Without symptoms } \\
\qquad(\mathrm{n}=58)\end{array}$ & P value \\
\hline Age (years) & 88 [IQR: 83-92] & 88 [IQR: 83-92] & 87 [IQR: 83-91] & 0.64 \\
\hline Gender (female, n, \%) & $127(84.7)$ & $74(80.4)$ & $53(91.4)$ & 0.061 \\
\hline Hypertension (n, \%) & $92(61.3)$ & $57(62.0)$ & $35(60.3)$ & 0.84 \\
\hline Diabetes $(\mathrm{n}, \%)$ & 28 (18.7) & $16(17.4)$ & $12(20.7)$ & 0.62 \\
\hline Heart failure (n, \%) & $12(8.0)$ & $6(8.7)$ & $4(6.9)$ & 0.70 \\
\hline Coronary heart disease (n, \%) & $25(16.7)$ & $16(17.4)$ & $9(15.5)$ & 0.76 \\
\hline Other heart diseases (n, \%) & $41(27.3)$ & $27(29.3)$ & $14(24.1)$ & 0.48 \\
\hline Atrial fibrillation $(\mathrm{n}, \%)$ & 28 (18.7) & $16(17.4)$ & $12(20.7)$ & 0.62 \\
\hline CKD $(n, \%)$ & $35(23.3)$ & $24(26.1)$ & $11(19.0)$ & 0.31 \\
\hline Stroke $(\mathrm{n}, \%)$ & $44(29.3)$ & $27(29.3)$ & $17(29.3)$ & 0.99 \\
\hline PTE $(n, \%)$ & $6(4.0)$ & $4(4.4)$ & $2(3.5)$ & 0.78 \\
\hline $\operatorname{COPD}(n, \%)$ & $13(8.7)$ & $11(12.0)$ & $2(3.5)$ & 0.055 \\
\hline Anemia (n, \%) & $15(10.0)$ & $9(9.8)$ & $6(10.3)$ & 0.91 \\
\hline Positive NP swab & $94(62.7)$ & $61(66.3)$ & $33(56.9)$ & 0.27 \\
\hline Positive LUS & $98(65.3)$ & $67(72.8)$ & $31(53.4)$ & 0.016 \\
\hline $\mathrm{Sa} O 2(\%)$ & $94.5 \pm 3.4$ & $93.5 \pm 3.5$ & $96.8 \pm 1.1$ & $<0.0001$ \\
\hline $\mathrm{Sa} \mathrm{O} 2<95 \%$ & $55(36.7)$ & $52(56.5)$ & $3(5.2)$ & $<0.0001$ \\
\hline
\end{tabular}

CKD, chronic kidney disease; COPD, chronic obstructive pulmonary disease; IQR, interquartile range; LUS, lung ultrasound; NP, nasal-pharyngeal; PTE, pulmonary thrombo embolism; Sa O2, oxygen saturation.

Table 2. Sensitivity, specificity, positive predictive value and negative predictive value of lung ultrasound, symptoms and oxygen saturation in predicting positivity of COVID-19 nasal-pharyngeal swabs.

\begin{tabular}{lllllllll} 
& TP & TN & FP & FN & Sensitivity & Specificity & PPV & NPV \\
Symptoms & 64 & 25 & 28 & 33 & $66 \%$ & $47 \%$ & $70 \%$ & $43 \%$ \\
Sa 02< 95\% & 38 & 41 & 19 & 52 & $42 \%$ & $68 \%$ & $67 \%$ \\
\hline Positive LUS & 74 & 32 & 24 & 20 & $79 \%$ & $57 \%$ & $74 \%$ \\
\hline
\end{tabular}

FN, false negative; FP, false positive; LUS, lung ultrasound; NPV, negative predictive value; PPV, positive predictive value; $\mathrm{Sa}_{2}$, oxygen saturation; TN, true negative; TP, true positive.

Table 3. Sensitivity, specificity, positive predictive value and negative predictive value of lung ultrasound, and oxygen saturation in predicting positivity of COVID-19 nasal-pharyngeal swabs in older residents with symptoms and in those without symptoms.

\begin{tabular}{|c|c|c|c|c|c|c|c|c|}
\hline & TP & TN & FP & FN & Sensitivity & Specificity & PPV & NPI \\
\hline \multicolumn{9}{|c|}{ With symptoms } \\
\hline $\mathrm{Sa} \mathrm{O}_{2}<95 \%$ & 38 & 17 & 13 & 24 & $61 \%$ & $57 \%$ & $75 \%$ & $41 \%$ \\
\hline Positive LUS & 51 & 15 & 16 & 10 & $84 \%$ & $48 \%$ & $76 \%$ & $60 \%$ \\
\hline \multicolumn{9}{|c|}{ Without symptoms } \\
\hline Positive LUS & 23 & 17 & 8 & 10 & $70 \%$ & $68 \%$ & $74 \%$ & $63 \%$ \\
\hline
\end{tabular}

For abbreviations and acronyms see Table 2. 
COVID-19 pneumonia and are more vulnerable to serious complications, particularly those with multimorbidity. This seems to be due to the close interpersonal interactions among residents, and between residents and staff members. As currently there is no vaccination available to prevent SARS-CoV-2 infection, avoiding exposure is the single most important measure to prevent the spreading of the disease. Nevertheless, since as many as $15-20 \%$ of patients tested negative for the SARS-CoV-2 infection show false negative results $[12,13]$, transthoracic imaging techniques should be suggested as tools to complement the use of rhyno-pharyngeal testing.

Thoracic imaging with chest-X ray and computed tomography are key tools for pulmonary disease diagnosis and management $[14,15]$, but their use is not practicable with the nursing home residents. LUS findings in COVID-19 pneumonia consist of laserlike, hyperechoic comet-tail artifacts arising from the pleural line, known as B-lines, moving synchronously with lung sliding, that depend on histopathologic changes characterized by alveolar edema with prominent proteinaceous exudates, vascular congestion, patchy inflammatory clusters with fibrinoid material, alveolar hyperplasia, and fibroblastic proliferation, that can help to differentiate COVID-19 pneumonia from other causes of respiratory disease [16-18]. The use of portable, wireless, pocket-sized scanners have allowed to execute LUS earlier and more easily [19]. Therefore, early assessment of LUS could be a valuable approach to investigate presence of COVID-19 lung involvement not only in hospitalized patients, including those of intensive care units, emer- gency departments, and internal medicine wards, but also in outpatient settings and in the community.

The results of our study show that LUS can be usefully performed in older patients with frailty, like those living in nursing homes. Our data outlined the necessity to prioritize the need to perform transthoracic echo scanning in older patients with COVID-19 lung injury with symptoms or in those that have been exposed to the contagion to improve clinical-decision making and to optimize measures of care and protection.

Since sensitivity of laboratory testing is of critical importance to avoid false negative results, combining transthoracic LUS imaging with SARS-CoV-2 nasal-pharyngeal sampling appears a valuable approach to identify patients with the disease. Patients showing signs of lung injury at LUS albeit tested negative for SARSCoV-2 infection at nasal-pharyngeal swabs comprised either patients suffering from the ongoing illness or those who have recovered from the disease but still display presence of lung involvement. Integration with clinical data will help to differentiate the two categories. Based on our data, we designed an algorithm to integrate the use of LUS with rhyno-pharyngeal swabs in patients with symptoms compatible with COVID-19 pneumonia and in apparently healthy subjects that have been exposed to sick individuals to predict the presence of the disease (Figure 2).

It is important to note that the integration of clinical evaluation, LUS, and swab testing allows proper decisions in terms of personalized treatment, need of isolating (or cohorting) positive patients to prevent further infection spreading, necessity of further diagnos-

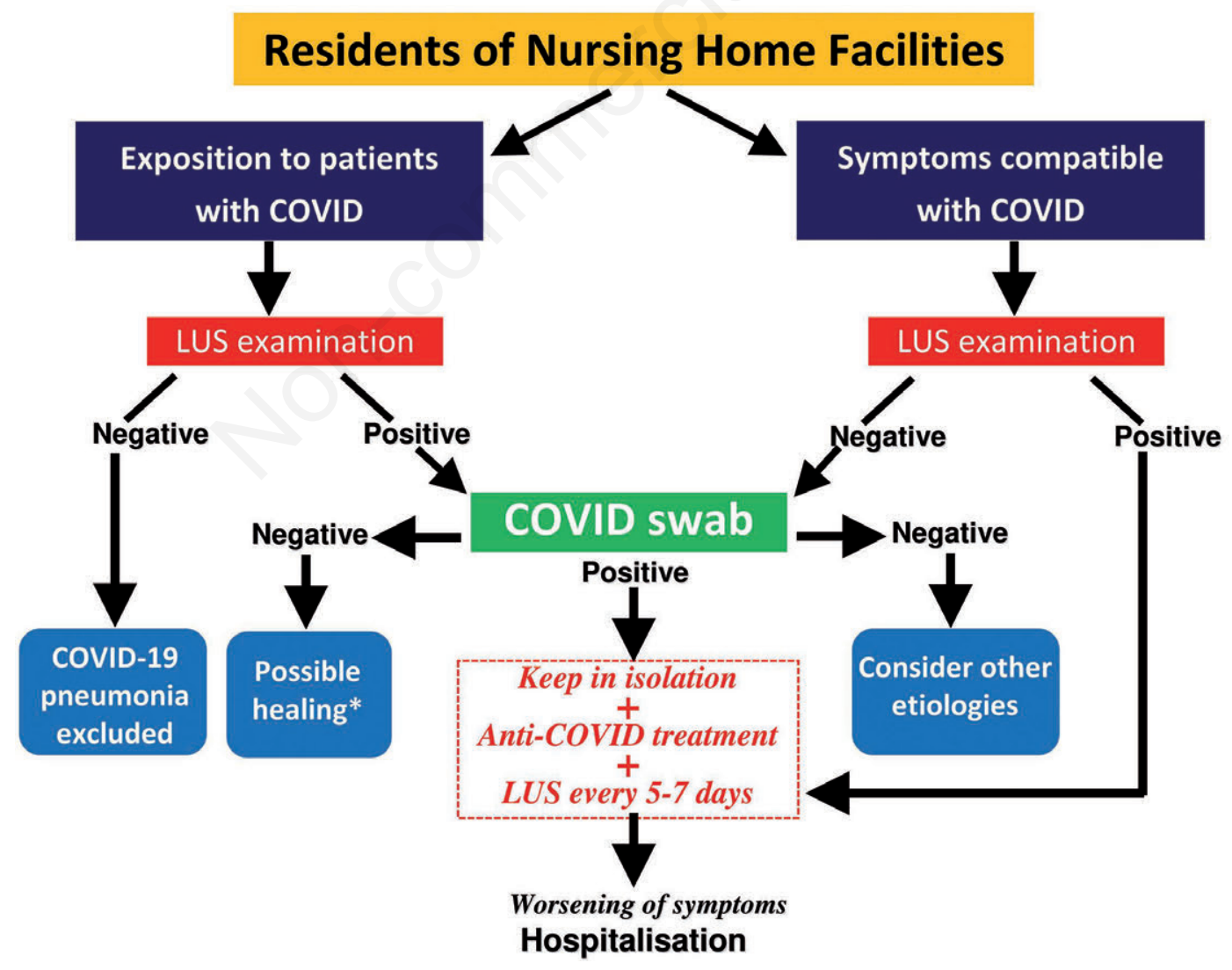

Figure 2. Flow-chart of intervention in residents of nursing homes with symptoms compatible to COVID-19 infection and in those exposed to the contagion of patients carrying the disease. ${ }^{*}$, to confirm the hypothesis of healing, rapid sierological test should be performed to detect the presence of IgG and IgM. 
tic steps either on-site or by transferring patients to the local emergency department $[9,20]$. This appears particularly relevant in the setting of pandemics like the SARS-CoV-2 infection, in which several nations reported the particularly unmet need of healthcare delivery in the nursing home facilities.

Several study limitations must be acknowledged. First, other conditions may be responsible of LUS alterations, including respiratory and cardiac diseases [21-23]. Second, although most protocols require to perform LUS over 12-14 zones, only a minority of our population underwent LUS in 12 zones, because many of them were too sick to undergo LUS in their posterior zones. Third, we assessed LUS only at one point in time, while follow-up evaluations have to be preferred especially for their prognostic implications [24]. Obviously, there are intrinsic limitations in a wireless system, as compared with other stand-alone machines with different probes, that have to be weighed in comparison with the necessity of portability in the nursing home setting. Echocardiography was not performed even though the assessment of right ventricular function would be valuable in older patients with COVID-19 pneumonia [25]. Finally, the reproducibility of the LUS technique was not evaluated in the study patients, even though reproducibility of transthoracic LUS has been previously fully explored [26].

In conclusion, LUS with portable wireless scanner echography allows bedside examination of as an extension of physical examination to diagnose presence of COVID 19-related lung injury in senior residents of nursing homes with symptoms and in those who have been exposed to the contagion. Thus, wireless LUS represents a valuable approach to reduce the risk of transmission of the infection and to prevent the spreading of the disease and, possibly, to decrease the high mortality risk of older adults living in senior living facilities. It is though critically important to combine LUS with nasal-pharyngeal swabs to improve sensitivity of diagnosis COVID-19 pneumonia or to simply support physical examination in case of a shortage of testing kits or their reagents.

\section{References}

1. Wang D, Yin $\mathrm{Y}, \mathrm{Hu} \mathrm{C}$, et al. Clinical course and outcome of 107 patients infected with the novel coronavirus, SARS-CoV2, discharged from two hospitals in Wuhan, China. Crit Care 2020;24:188.

2. Guan WJ, Ni ZY, Hu Y, et al. China Medical Treatment Expert Group for Covid-19. Clinical Characteristics of Coronavirus Disease 2019 in China. N Engl J Med 2020;382:1708-1720.

3. Garier-Crussard A, Foretier E, Gilbert T, et al. Novel Coronavirus (COVID-19) epidemics: What are the risks for older patients? J Am Geriatr Soc 2020;68:939-40.

4. Applegate WB, Ouslander JG. COVID-19 presents high risk to older persons. J Am Geriatr Soc 2020;68:681.

5. Shahid Z, Kalayanamitra R, McClafferty B, et al. COVID-19 and older adults: What we know. J Am Geriatr Soc 2020;68: 926-9.

6. Sun H, Ning R, Tao Y, et al. Risk factors for mortality in 244 older adults with COVID-19 in Wuhan, China: a retrospective study. J Am Geriatr Soc 2020;68:E19-E23.

7. Malone ML, Hogan TM, Perry A, et al. COVID-19 in older adults: key points for emergency department providers. J Geriatr Emerg Med [Epub haed of print].

8. Mojoli F, Bouhemad B, Mongodi S, Lichtenstein D. Lung ultrasound for critically ill patients. Am J Respir Crit Care Med 2020;199:701-714.

9. Soldati G, Smargiassi A, Inchingolo R, et al. Proposal for international standardization of the use of lung ultrasound for patients with COVID-19: A simple, quantitative, reproducible method. J Ultrasound Med 2020;39:1413-9.

10. Vermeiren C, Marchand-Senécal X, Sheldrake E, et al. Comparison of Copan Eswab and FLOQswab for COVID-19 PCR diagnosis: working around a supply shortage. J Clin Microbiol 2020;58:e0669-20.

11. Landis, J, Koch G. The measurement of observer agreement for categorical data. Biometrics 1977;33:159-74.

12. Sharfstein JM, Becker SJ, Mello MM. Diagnostic testing for the novel Coronavirus. JAMA 2020 [Epub haed of print].

13. West CP, Montori VM, Sampathkumar P. COVID-19 testing: the threat of false negative results. Mayo Clin Proc 2020;10.1001/jama.2020.3864.

14. Rubin GD, Ryerson CJ, Haramati LB, et al.. The Role of Chest Imaging in Patient Management during the COVID-19 Pandemic: A Multinational Consensus Statement from the Fleischner Society. Chest 2020;158:106-16.

15. Lei J, Li J, Li X, Qi X. CT imaging of the 2019 novel coronavirus (2019-nCov) pneumonia. Radiology 2020;295:18.

16. Soldati G, Smargiassi A, Inchingolo R, et al. Is there a role for lung ultrasound during the COVID-19 pandemic? J Ultrasound Med 2020;39:1459-62.

17. Volpicelli G, Lamorte A, Villén T. What's new in lung ultrasound during the COVID-19 pandemic. Intensive Care Med 2020;46:1455-8

18. Peng O, Wang X, Zhang L. Findings of lung ultrasonography of novel Coronavirus pneumonia during the 2019-2020 epidemic. Intensive Care Med 2020;46:849-50.

19. Smith MJ, Hayward SA, Innes SM, Miller ASC. Point-of-care lung ultrasound in patients with COVID-19 - a narrative review. Anaesthesia 2020;75:1096-104.

20. Liu RB, Tayal VS, Panebianco NL, et al. Ultrasound on the Frontlines of COVID-19: Report from an International Webinar. Acad Emerg Med 2020;27:523-6.

21. Ye X, Xiao H, Chen B, Zhang S. Accuracy of lung ultrasonography versus chest radiography for the diagnosis of adult community acquired pneumonia: review of the literature and metaanalysis. Plos One 2015;10:e0130066.

22. Soldati G, Demi M. The use of lung ultrasound images for the differential diagnosis of pulmonary and cardiac interstitial pathology. J Ultrasound 2017;20:91-96.

23. Gargani L, Pang PS, Frassi F, et al. Persistent pulmonary congestion before discharge predicts rehospitalization in heart failure: a lung ultrasound study. Cardiovasc Ultrasound 2015;13:40.

24. Pugliese NR, Fabiani I, Conte L, et al. Persistent congestion, renal dysfunction, and inflammatory cytokines in acute heart failure: a prognosis study. J Cardiovasc Med (Hargerstown) 2020;21:494-502.

25. Rosa GM, D’Agostino A, Giovinazzo S, et al. Echocardiography of right ventricular arterial-coupling predicts survival of elderly patients with heart failure and reduced to mid-range ejection fraction. Monaldi Arch Chest Dis 2020;90:10.4081/monaldi.2020.1269.

26. Anderson K, Fields J, Panebianco N, et al. Inter-rater reliability of quantifying pleural B-lines using multiple counting methods. J Ultrasound Med 2013;32:115-20. 